\title{
Coordinadoras culturales: formaciones transversales en Chile durante la dictadura*
}

CULTURAL COORDINATORS: TRANSVERSAL TRAINING IN CHILE DURING THE DICTATORSHIP

COORDENADORAS CULTURAIS: TREINAMENTO TRANSVERSAL NO CHILE DURANTE A DITADURA

\section{Paulina E. Varas** Javiera Manzi A.***}

Cuadernos de Música, Artes Visuales y Artes Escénicas

/ Volumen 14 - Número 2 / Julio - Diciembre de 2019

/ ISSN 1794-6670 / Bogotá, D.C., Colombia / pp. 55-74

Fecha de recepción: 31 de diciembre de 2018

Fecha de aceptación: 19 de febrero de 2019

Disponible en línea: 25 de junio de 2019

doi 10.11144/javeriana.mavae14-2.ccft

* $\quad$ Artículo de investigación. Este proyecto forma parte de la Investigación "Coordinadoras culturales en dictadura", financiada por el FONDART de investigación en Artes Visuales 2019.

* $\quad$ Doctora en Historia y Teoría del Arte por la Universidad de Barcelona. Académica e investigadora de la Universidad Andrés Bello. Co-coordina de CRAC Valparaíso, una plataforma de pensamiento y célula de acción crítica. Miembro de la Red Conceptualismos del Sur y del equipo de investigación The Force of art.

*** A research in a global perspective (2019-2020), Prince Claus Foundation. ORCID 0000-0002-5641-7724.

Socióloga y archivera por la Universidad de Chile, curadora, docente e investigadora independiente. Investiga las líneas de relación entre arte, política y redes de solidaridad. Actualmente es coordinadora de la Red Conceptualismos del Sur e integrante del Centro Social y Librería Proyección. ORCID 0000-0002-9390-0002

\section{Cómo citar:}

Varas, Paulina E. y Javiera Manzi A. 2019. "Coordinadoras culturales: formaciones transversales en Chile durante la dictadura". Cuadernos de Música, Artes Visuales y Artes Escénicas 14 (2): 55-74. http://doi.org/10.11144/javeriana.mavae14-2.ccft 


\section{Resumen}

El presente artículo se enfoca en la experiencia que tuvieron en Chile durante la dictadura cívico-militar las coordinadoras culturales. En el escenario represivo, junto con el clima de censura que se vivía en la época, emergieron formas de articulación colectiva y transversal que vincularon a diferentes agrupaciones y organizaciones del mundo del arte, la cultura, la defensa a los derechos humanos, sindicatos, grupos de poblaciones y parroquias, para sostener las iniciativas culturales y la lucha antidictatorial que coexistía en ese momento. Los objetivos de esta investigación son contextualizar estas experiencias y entregar información valiosa que permita comprender los tipos de organizaciones que participaron, así como algunas de las actividades que se desarrollaron a lo largo de estos diecisiete años. Esta investigación se realizó a partir de archivos y colecciones particulares que algunos artistas chilenos han conservado. Se ha llevado a cabo un análisis documental de este material, así como la utilización de conceptos teóricos para abordar críticamente tales materiales. Las experiencias de las coordinadoras culturales analizadas en el artículo no presentan estudios precedentes y han sido muy poco referenciadas en el escenario nacional, así, este artículo quiere contribuir con este vacío documental del periodo.

Palabras clave: coordinadoras culturales; apagón cultural; resistencia; dictadura; arte; transversalidad.

\section{Abstract}

This paper focuses on the experience of cultural coordinators in Chile during the civic-military dictatorship. During the repressive scenario, along with the climate of censorship of the time, different forms of collective and transversal articulation emerged that were linked to different groups and organizations from the world of art, culture, defense of human rights, trade unions, groups of populations and parishes, to sustain cultural initiatives and the struggle against dictatorship that coexisted at that time. The objectives of this research are to contextualize these experiences and to provide valuable information that allows us to understand the types of organizations that participated, as well as some of the activities carried over these seventeen years. This research was carried out based on the archives and private collections kept by a number of Chilean artists. A documentary analysis of this material was carried out, as well as the use of theoretical concepts to critically address those materials. The experiences of cultural coordinators analyzed in the paper have not been previously studied, and little reference has been made to them in the national scenario; as such, this article aims to contribute to this documentary gap of the period.

Keywords: cultural coordinators; cultural blackout; resistance; dictatorship; art; transversality.

\section{Resumo}

Este artigo foca na experiência que as coordenadoras culturais tiveram no Chile durante a ditadura cívico-militar. No cenário repressivo, junto com o clima de censura vivido na época, surgiram formas de articulação coletiva e transversal que articularam diferentes grupos e organizações do mundo da arte, a cultura, a defesa dos direitos humanos, os sindicatos, os grupos populacionais e as paróquias, para sustentar as iniciativas culturais e a luta contra a ditadura que coexistia na época. Os objetivos desta pesquisa são contextualizar essas experiências e fornecer informações valiosas que permita entender os tipos de organizações que participaram, bem como algumas das atividades desenvolvidas ao longo desses dezessete anos. Esta investigação foi feita a partir de arquivos e coleções particulares que alguns artistas chilenos preservaram. Uma análise documental deste material foi realizada, bem como o uso de conceitos teóricos para abordar criticamente esses materiais. As experiências das coordenadoras culturais analisadas no artigo não apresentam estudos prévios e têm sido pouco referenciadas no cenário nacional; portanto, este artigo pretende contribuir com esta brecha documentária do período.

Palavras-chave: coordenadoras culturais; blecaute cultural; resistência; ditadura; arte; transversalidade. 


\section{INTRODUCCIÓN}

La historia de las prácticas artísticas y culturales durante el periodo dictatorial en Chile (1973-1989)' ha sido objetivo de diversos y vastos estudios que demarcan la constitución de distintas escenas y campos. En estos prima una mirada que escinde y remarca las disputas y los quiebres entre agentes y grupos de distintos sectores (Aguiló 1983; Brunner 1985; Richard 1986) que construyeron sus trayectorias críticas desde la diferenciación. Sin desconocer esta producción de diferencias, el presente estudio se centra en las experiencias de transversalidad que se configuraron a través de las coordinadoras culturales del periodo dictatorial, las que aglutinaron a distintos actores del mundo de la cultura de oposición con otros sectores, con lo que ampliaron el ámbito de intervención y enlazamiento contrahegemónico.

Uno de los aspectos más desconocidos de este periodo son las redes y articulaciones entre artistas, escritores, teatristas, folcloristas, muralistas, organizaciones de derechos humanos, sindicatos, gráficos, audiovisualistas y músicos que dieron lugar a un entramado complejo, heterogéneo y sumamente activo en las coordinadoras culturales. Estas desempeñaron un papel sumamente significativo en la regeneración del tejido social a partir de la conformación de redes de colaboración, solidaridad y creación colectiva transversales al mundo del arte y la cultura de oposición a la dictadura. A diferencia de otros estudios del periodo que se centran en la fragmentación del campo cultural, este artículo busca evidenciar la existencia de entramados amplios y heterogéneos del que participaron una multiplicidad de actores del mundo de la cultura, las organizaciones sociales y los grupos militantes, donde las bases no se sostenían desde el punto de vista de un consenso en el que prevalecía un solo punto de vista, sino que se definen desde su propio disenso heterogéneo e integrador de las diferencias.

Las tres principales coordinadoras culturales de este periodo fueron la Unión Nacional por la Cultura (UNAC) fundada en 1977, el Coordinador Cultural (CC) fundado en 1983 y la Coordinadora de Gremios del Arte (CGA) fundada en 1985. Así, contra la idea establecida de que durante la dictadura se vivió en un "apagón cultural" (Donoso 2013, 109) donde prevaleció la censura sobre los medios de comunicación, la intervención en universidades con docentes exonerados y estudiantes expulsados, el cierre del espacio público y un "golpe estético" (Errázuriz y Leiva, 2012) sobre la vida social y cultural del país, nos resulta fundamental indagar estas otras historias y memorias que no han sido visibilizadas, y así contribuir a recrear formas instituyentes en la cultura y en las artes. ${ }^{2}$ Cabe señalar que uno de los aspectos más notables de la invisibilización del rol y de la presencia de las coordinadoras culturales durante este periodo es el desconocimiento respecto de la diversidad de artistas visuales ampliamente reconocidos del periodo que formaron parte de ellas $y$, en especial, del lugar de las mujeres como agentes movilizadoras.

Este artículo es un primer resultado de un proceso de investigación ${ }^{3}$ en curso en que hemos desarrollado una aproximación situada e interdisciplinaria en la que se entremezcla la sociología con la teoría e historia del arte. Nos interesa esta síntesis teórico-metodológica que posibilita un diálogo en torno a los entramados y las trayectorias críticas de las coordinadoras culturales. La pertinencia de este entrelazamiento se explica por la necesidad de concebir algunas experiencias de articulación colectiva en su complejidad social y estético política, así como ahondar en sus espacios de agenciamientos colectivos y micropolíticos. 
Para la elaboración de esta investigación, se contempló un proceso de revisión documental en archivos y colecciones particulares de artistas quienes no solo formaron parte de instancias, sino también cuidaron sus documentos y realizaron registros escritos y sonoros de estos. El material incluido en los acervos de Luz Donoso,Virginia Errázurriz, Lilia Santos, Antonio Kadima, Cucho Márquez y Havilio Pérez, incluye actas, correspondencia, afiches, panfletos, publicaciones, casetes de audio, videos y fotografías.

Este artículo esta dividido en tres momentos. El primero presenta una contextualización del escenario cultural chileno en la dictadura y se enfoca en el denominado "apagón cultural", noción que intentaba neutralizar el escenario de efervescencia muchas veces subterránea que tuvieron las coordinadoras culturales, las organizaciones y los grupos culturales durante el régimen represivo. Luego, un en un segundo momento, se aporta una serie de conceptualizaciones teóricas sobre este tipo de articulaciones culturales desde una perspectiva éticopolítica como parte del marco de comprensión de estas prácticas. Finalmente, se presenta un primer acercamiento a la experiencia de las tres principales coordinadoras culturales del periodo dictatorial en Chile.

\section{QUIEBRES Y CONTINUIDADES DEL CAMPO CULTURAL TRAS EL GOLPE DE ESTADO}

Tras el golpe de Estado en 1973 a cargo de la Junta Militar que acabó con el Gobierno de la Unidad Popular (UP), se interrumpe un ciclo de desarrollo cultural vinculado estrechamente a las políticas de democratización y socialización del Estado. El quiebre institucional que esto trajo consigo impactó profundamente el mundo de la cultura tanto en los espacios hegemónicos vinculados a la Universidad de Chile y al Museo Nacional de Bellas Artes como en la amplia gama de expresiones y prácticas culturales venidas desde sectores populares. En ambos casos, lo que comienza a operar son, como plantea tempranamente Brunner (1979), "Ios cuatro conjuntos de políticas del modelo cultural del autoritarismo: políticas de exclusión (represión de los agentes disidentes), políticas de control (clausura del espacio público), políticas de regulación (por medio del mercado) y políticas de producción (ideológico-cultural)" (5). Si nos ceñimos a esta distinción, podemos observar las políticas de exclusión particularmente en el periodo de los años más represivos en la instalación del régimen, 1973-1975, cuando la exclusión no es otra cosa que la eliminación física mediante la ejecución, la desaparición, el encarcelamiento, el destierro y el exilio de militantes, artistas, intelectuales, pobladores, sindicalistas, estudiantes, académicos (estos dos últimos, sujetos de exoneraciones masivas) asociados a la UP. Junto con lo anterior, un segundo ámbito de exclusión remite a la violencia simbólica del régimen que está asociado a lo que Errázuriz y Leiva (2012) denominaron "operación limpieza" dentro del "golpe estético de la dictadura", que "representa, por una parte, la 'desinfección' del pasado marxista y, por otra, la promoción de una noción militarizada de la estética cotidiana, que se refleja en rasgos tales como la depuración, el orden y la restauración fervorosa de los símbolos patrios" (15). Así es como las políticas de eliminación por medio del desmantelamiento de museos, universidades y centros culturales, la quema de libros, la persecución a todo material gráfico considerado subversivo (Cristi y Manzi 2016) y el borramiento de murales realizados por las brigadas muralistas, la censura sobre todo signo crítico y el robo del 
acervo cultural en instituciones intervenidas se fundaron en el imperativo de extirpación y quiebre con todos los indicios que señalaran la persistencia del proyecto de la vía chilena al socialismo, así como con la emergencia de grupos de resistencia. Vinculado a esto mismo, las políticas de control expresadas en la clausura del espacio público significaron la retirada forzada de todas las iniciativas político-culturales que solían desplegarse en las calles, plazas o instituciones públicas, para quedar sometidas a la clandestinidad o a lugares que debían poder sortear la vigilancia.

La contracara de la eliminación y persecución de todo signo que remitiera al pasado reciente o a una construcción crítica desde las artes y la cultura fue la instalación de una cultura oficial ("políticas de producción ideológico-cultural") en la que primó, por una parte, la impronta nacionalista que reivindicaba el cuño hacendal y miliciano como signos de chilenidad, y por otra, los efectos de la implantación del modelo neoliberal a partir del influjo del comercio internacional, la ola de privatizaciones de industrias y bienes públicos y el "boom publicitario" (Álvarez 2004, 143) ("políticas de regulación por medio del mercado"), que transformaron el repertorio de la vida social y cultural del país.

\section{¿APAGÓN CULTURAL? DERIVAS DE LA RELACIÓN ENTRE ARTE Y CAMPO CULTURAL EN DICTADURA}

Una de las estrategias de denuncia de la situación cultural del país en dictadura fue acuñada en la expresión "apagón cultural" (Donoso 2013, 109), tal como fue utilizada en el contexto de las Jornadas del Libro y la Cultura de 1977 para referirse a la disminución en las publicaciones del mundo editorial y que luego fue asumida desde sectores de izquierda, en especial desde el exilio para impugnar la totalidad del quiebre cultural institucional y extrainstitucional. En este punto, la investigadora Karen Donoso presenta una genealogía interesante del término, al advertir que, paradójicamente, sus primeros usos están vinculados precisamente al régimen, puesto que fue intencionado como una crítica al "apagón intelectual" que trajo la UP "a partir de una 'crisis educativa' en la cual estaría inmersa la juventud chilena, producto del 'proceso de politización' que vivió el país en las décadas anteriores" (Donoso 2013, 109) y, luego, fuera reivindicado por el asesor cultural de Pinochet, Enrique Campos Menéndez, como una realidad superada por la oferta cultural oficialista.

No obstante, la referencia al "apagón cultural" aparece continuamente en los testimonios de artistas y trabajadores culturales del periodo, en la mayoría de los casos a modo de denuncia al régimen, pero en otros como un cuestionamiento a esta noción en tanto tiende a negar la existencia del campo cultural y artístico no oficialista que existió a contrapelo de la cultura dominante. Frente a esto, "desmitificar el apagón implica oponerse a la invisibilización que aquel provocó entonces y que sigue provocando cada vez que se impone como una matriz interpretativa, pues obstruye la potencia de una memoria de ebulliciones permanentes y descentradas que paulatinamente dieron forma a la trama de una cultura subterránea que la dictadura no pudo apagar" (Cristi y Manzi 2016, 50). Lejos de la idea de "apagón", la alternativa a la "cultura autoritaria" se desarrolló a lo largo de los diecisiete años de dictadura, de diferentes maneras según el momento político y social en que se desenvolvió tanto el régimen como la oposición. De acuerdo con los análisis realizados por Aguiló (1983) y Rivera (1983), es posible distinguir tres fases del desarrollo político cultural en dictadura. 
Un primer momento de negación radical (1973-1977) que en el espacio popular se presentará como un ciclo de repliegue y añoranza cultural en el que se multiplicarán espacios tales como peñas folclóricas y talleres culturales populares en tanto estrategias de recomposición del tejido social y restauración de un patrimonio cultural vedado.

Un segundo momento (1977-1983), Ilamado "la etapa fundacional" de la dictadura cívico-militar, operó a nivel cultural bajo "medidas encaminadas a perfilar más definitivamente el nuevo modelo económico, sus líneas sociales políticas y culturales" (Aguiló 1983, 15). Es en este periodo cuando se impone la Constitución de 1980, junto con una serie de reformas que transformaron el modelo de desarrollo, cuyas crisis darán inicio a movimientos cada vez más públicos de oposición y resistencia, tal es el caso de las huelgas de trabajadores que llevarán al primer paro nacional contra el Plan Laboral de 1979. En palabras de Aguiló,

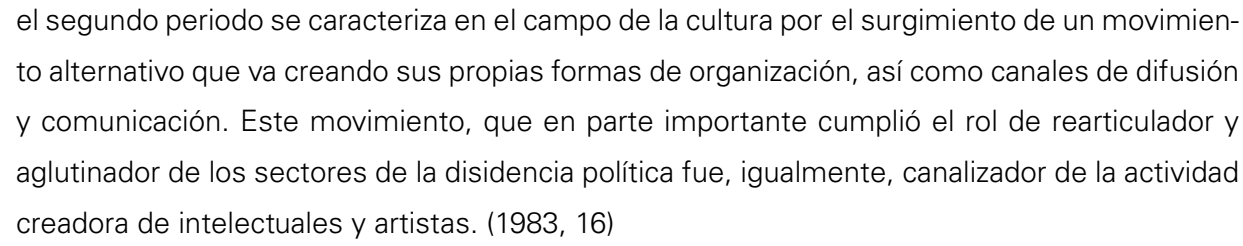

En este contexto, irrumpe la actividad pública de los frentes culturales y, paralelamente, la emergencia desde 1977 del campo de prácticas artísticas y escriturales que Richard (1986) reuniría bajo lo que designa como "escena de avanzada".

Finalmente, un tercer momento es posible datarlo desde 1983 con el inicio de las Jornadas de Protesta Nacional que abre un ciclo de movilizaciones y politización que da lugar al despliegue de una cultura rebelde de nuevo cuño. En este último, la crítica política coincide con nuevos referentes generacionales de mayor irreverencia y desacato a la cultura tradicional de izquierda, que a su vez se entrecruzan en el despliegue crítico de los feminismos, de las disidencias sexuales y de los circuitos under. Cabe señalar, en relación con este último periodo, que es posible distinguir una fase final, que corresponde al periodo de 1986 a 1989, cuando, tras el fin del ciclo que abren las Jornadas de Protesta Nacional y la derrota de lo que fue concebido como el año decisivo en la lucha contra la dictadura, se abre un momento final dirigido por ciertos sectores a preparar y proyectar el proceso transicional.

\section{ESCRITURAS CRÍTICAS SOBRE EL CAMPO DE LAS ARTES VISUALES EN DICTADURA}

En el campo de las artes visuales, Richard (1986) analiza la coyuntura sociopolítica y elabora un relato referido a lo que denomina "escena de avanzada" en que señala: "Esta designación es primeramente operativa: nos ha permitido, durante estos años en Chile, nombrar el trabajo de creadores empeñados en reformular las mecánicas de producción artística y de lenguaje creativo, en el marco de una práctica contrainstitucional" (20). Es necesario para esta investigación debatir la periodificación establecida por Richard, quien establece un corte temporal desde el golpe militar y las prácticas artísticas precedentes prácticamente sin continuidad, toda vez que esta investigación propone hacer cruces temporales más directos 
y continuos según experiencias culturales que construyeron formas de coordinación colectiva con el fin de realizar acciones culturales para la restitución de la democracia junto con la producción de un importante acervo testimonial de pensamiento crítico sobre las condiciones de artistas y trabajadores culturales del periodo dictatorial. Para Richard (1986), la emergencia de la escena de avanzada marca un precedente fundacional que a la vez indicaba la discontinuidad con otros grupos y tradiciones artísticas de la oposición, puesto que,

la experiencia contestataria y protestararia de un arte del testimonio y la denuncia prevalece sobre cualquier exigencia formal de redefinición crítica de las pautas de lenguaje, ya que la cultura militante considera que las rupturas de códigos amenazarían con estropear el precario consenso de identidad que debieron retejer los excluidos y los perseguidos en medio de la adversidad nacional [a diferencia del énfasis crítico-experimental de la escena de avanzada]. (129)

Richard (1986) estableció también una diferencia entre las organizaciones militantes de la cultura opositora al régimen militar y los artistas de la escena de avanzada, aunque a todos los vinculaba la contraposición al régimen militar, pero los últimos se situaban en una "marginalidad polémica" con las organizaciones militantes.

Aguiló (1983) propone que a fines de 1975, en un concurso en el Museo Nacional de Bellas Artes de Santiago, se redefinen las formas de enfrentar las condiciones socioculturales del periodo dictatorial. Por un lado, artistas que se abocaron a técnicas tradicionales, pero sin una preocupación rigurosa y artistas con "un trabajo al margen de las instituciones, en una búsqueda por fundar teóricamente un ansia de inscripción cultural, acompañada por el uso experimental de técnicas mixtas" (14). Si bien Aguiló se refiere más específicamente a artes visuales, realiza un análisis del campo cultural donde aparecen mencionadas iniciativas contraculturales, sobre todo, espacios de encuentro extrainstitucionales para creadores y artistas.

Las coordinadoras culturales emergieron en distintos momentos del periodo dictatorial, lo que definió las continuidades y rupturas con el campo artístico anterior al golpe, los grupos que formaron parte de su entramado, el rol que cumplieron y las formas de relación entre arte y política que se establecieron en cada caso. Las tres generaciones de coordinadoras impulsaron acciones que formaron parte de un campo artístico y cultural de oposición a la dictadura que fue no solo un espacio de resistencia, sino también de creación de alternativa. Estas instancias fueron experiencias asociativas, espacios de difusión, experimentación y de producción artística que retomaron la solidaridad y la acción colectiva como los principios activos de la presencia de un arte negado en el espacio social.

\section{MICROPOLIITICA Y TRANSVERSALIDAD}

Pensar las prácticas culturales como prácticas transversales implica pensarlas como posibilidades y potencias, y cómo estas mismas advienen actuales. La transversalidad es una multiplicidad de líneas de fuga, como señalan Gilles Deleuze y Félix Guattari (1980), es una capacidad de sortear cercos de todo tipo, lo que implica entrar en coeficientes de libertad. Se trata de un movimiento que está habilitado por un tipo de plasticidad o elasticidad subjetiva y cognitiva; es poder darle acceso a un imaginario propio. La transversalidad se sitúa de 
manera adyacente a los círculos cerrados que establecen un mismo objetivo que se persigue de manera consensuada. No se trata de ir al colapso o al enfrentamiento, sino de zambullirse allí mismo y resistir; se trata de una oda a la vida que no participa del etnocidio; es un canto a la vida, tal como decía Violeta Parra. Desde esta perspectiva, "la resistencia no consiste en una mera reacción a la dominación, sino que la resistencia y la insurrección, como conceptos antidialécticos, son productivos, afirmativos, creativos" (Raunig 2007, 5), crean posibilidad de entrecruces allí donde el deseo emerge como potencia creadora de mundos.

Los regímenes dictatoriales más estriados o jerárquicos tienen grietas, por allí se deslizan prácticas micropolíticas que "devienen fluidos" en tanto dejan lo identitario que en sí mismo es un cierre por el devenir que es un proceso. La multiplicidad es entendida acá como la coexistencia de heterogeneidades, considerando que una misma ya es de por sí una multiplicidad en la que emerge lo disensual que es también una multiplicidad de valores, vertientes y posibilidades; al implicarse en un proyecto colectivo, "uno lleva también su historia, su cultura, su lengua, sus relaciones con los poderes y los aprendizajes, sus fantasmas y sus deseos. Estos no son, propiamente hablando, individuales o privados, sino que se inscriben en una multitud de relaciones geográficas, sociales, económicas, familiares" (Vercauteren, Mouss y Müller 2010, 135); este problema micropolítico es vital para construir lo común de un grupo.

La transversalidad en los grupos en tanto líneas de fuga que fluyen en un desliz, que pasan entre las grietas, son también espacios precarios y frágiles, hay una fragilidad interna que también resiste a los acosos desde el exterior. Si se entiende que el éxito de estas experiencias estuviera determinado por su duración en el tiempo, sería también una trampa, pues muchas veces estas experiencias a partir de un determinado ciclo de duración se institucionalizan, por ejemplo, se crean experiencias dependientes del Estado o coalescentes al sistema. Toda escuela deviene su uso y permanencia como identitaria y excluyente. Comienzan las preguntas por quién dirige, quién será el jefe, se jerarquizan experiencias cuyas formas de organización internas provenían del deseo y de la necesidad de los vínculos, como señala Guattari: "El criterio de un buen grupo consiste en no soñarse único, inmortal y significante [...] sino en conectarse con un afuera que lo confronte con sus posibilidades de sinsentido, de muerte o de fragmentación, por la misma razón de su apertura a los demás grupos" (citado en Vercauteren et al. 2010, 53). Por ello, es que la fragilidad de estos espacios coexiste con el cuidado de sí en tanto la vulnerabilidad a la que está expuesta su permanencia en el tiempo se torna vital. El cuidado de sí se entrelaza al cuidado del grupo, que permite equiparse para los imprevistos. "Foucault define el cuidado de sí como la posibilidad de mantener relaciones y vínculos con otros, cuidar y velar por lo que se piensa y llevar a cabo acciones a través de las cuales uno se hace cargo de sí mismo, a través de las que uno se modifica" (citado en Vercauteren et al. 2010, 66).

Cuando el ciclo de estas experiencias finaliza, aparece una instancia operativa en la que se reanima este deseo como formas de creer en el mundo, de haber sido capaz de proteger y cuidar el deseo, y de inventar una vida a pesar de la infamia. En este sentido, emerge la transformación social; aunque la situación es adversa, se pueden seguir estableciendo vínculos, esto es, la micropolítica del cuidado, un mundo soñado deviene posible, la presencia del cuerpo, un cuerpo colectivo. 


\section{FORMACIONES DE OPOSICIÓN}

Williams (1994), a propósito de formaciones culturales, distingue entre tres tipos "en función de las relaciones declaradas y reales que los actores sociales establecen con otras organizaciones del mismo campo o de la sociedad en general": las de especialización, las alternativas y las de oposición (citado en Amigo et al. 2012, 78). Para Williams, las formaciones de especialización corresponden a aquellas en las que "se promueve un trabajo en un medio o rama particular del arte" (65), las alternativas a aquellas que "aportan medios alternativos para la producción, exposición o publicación de obras" (65) cuando las instituciones las excluyen y finalmente las formaciones de oposición a aquellas que "se convierten en una oposición activa frente a las instituciones establecidas, o de una manera más general, frente a las condiciones dentro de las cuales existen" (65). Estas últimas, por su carácter crítico y abiertamente confrontacional a la cultura y las instituciones dominantes, se acerca más claramente a la experiencia de organizaciones político-culturales que pretenden participar de un proceso de transformación de su medio y contexto general. Nos parece posible a la vez contemplar la existencia de formaciones híbridas, en las que la oposición coexista con la producción de alternativas autónomas o en las que la promoción de ciertos sectores culturales esté aparejado con un posicionamiento crítico frente al contexto general.

\section{COORDINADORAS CULTURALES}

Nos interesa pensar el papel de las coordinadoras culturales como una trama en la que no solo se reconozca su carácter múltiple y transversal, sino también su contribución en la regeneración y ampliación de vínculos y en la elaboración de nuevas poéticas y lenguajes críticos. De ahí la centralidad del relato sobre aquellas experiencias en que primó lo colectivo y lo cooperativo dentro de las prácticas artísticas y culturales del periodo.

\section{UNIÓN NACIONAL POR LA CULTURA}

La UNAC se funda en 1977 como la primera de estas instancias de articulación de coordinadoras culturales (figura 1).

A solo cuatro años de la dictadura, la situación nacional estaba impregnada de un clima de represión brutal, personas desaparecidas, instituciones públicas apropiadas e intervenidas como las universidades, toque de queda, persecución política, entre otros. Es en este clima en que emergió la necesidad y urgencia de las personas y agrupaciones que trabajaban en cultura de conformar modos de defender sus prácticas y también manifestar su disconformidad con el contexto represivo en vías de una defensa por la vida. La UNAC desarrolló una serie de acciones, llamados, informes, actividades públicas y encuentros de los diferentes grupos y comisiones que participaron. La idea principal fue la de unir y coordinar el trabajo de personas y agrupaciones de las diferentes áreas artísticas para constituir un frente que fuera expresión unitaria de intereses e inquietudes en el movimiento cultural. En ella participaron Sociedad de Escritores de Chile (SECH), Canto Nuestro, Taller 666, Taller Espacio, TAV, CADA, Revista Araucaria, Agrupación Cultural Universitaria (ACU), Asociación de Fotógrafos 
de Chile (AFI), Teatro ICTUS, Nuestro Canto, Agrupación Siglo XX, Taller Mingaco, Unión de Escritores Jóvenes, Sello Alerce, Federación de Cineastas, Agrupación de Músicos Jóvenes, Teatro Imagen, Comisión Chilena pro Derechos Juveniles (Codeju), Taller Contemporáneo, Asociación Organizaciones Juveniles, Organización Nacional de la Cultura y el Arte Infantil (Ocarin), Agrupación Cultural Santa María y Agrupación de Plásticos Jóvenes (APJ), entre otras. La UNAC estaba coordinada por un cuerpo directivo que daba forma y orientaba el trabajo y lo conformaban personas representantes de algunas de las organizaciones.

ENCUENTRO .NACIONAL "UNAC"

INFORME COMISION "LIBERTAD DE EXPRESION-LIBERTAD DE CREACION" AREA PLASTICA

(-Cómo enfrentar el problema de la libertad de expresión y creación, cuando no se es libre?)

\section{INITRODUCCION}

A partir de 1973, la libertad está conculcada en nuestro país. El pleno conocimiento de ésta situación - que afecta profundamen te el quehacer artístico y cultural -, nos mueve primeramente, a establecer una serie de hechos y realizar un sonero análisis de ellos; para luego plantear algunas proposiciones realistas, $-\mathrm{y}$ por ende posibles como primer punto para ir logrando un más amplio margen de expresión creadona y de participación.

Nos enfrentanos ante una realidad nueva para nosotros, a pesar de la información de experiencias similares que manejamos.

Ante ella, no henos sido capaces de generar án, nuestra propia y colectiva respuesta, la que nos permitiría acercarnos a la recuperacion de nuestros valores auténticos, y a futuro gestar un nue vo concepto creador.

Hoy, sin embargo,- pasados ya siete largos afios-tenemos más que suficientes elementos de conocimientos empírico, té́rico y vivencial, que nos obligan a asumix conscientemente nuestra responsabi lidad personal y colectivo, ante cl momento histórico que vivimos.

Pensamos que éste Encuentro Nacional por la Cultura que realiza la UNAC, es el punto de partida, que habrá de concretar nuestra voluntad de unidad y trabajo por la recuperación de nuestra liber tad creadora.

Nos encontramos ante graves y profundas modificaciones - impuestas por el xégimen-, en nuestro modo de convivencia nacional, 10 que condiciona y obliga a replantearnos el papel del creador en el Chile de hoy.

La realidad del creador actual, supone un margen de permanente violencia, riesgos y carencias, - que va desde 10 físico a lo inte lectual-y dentro del cual está innerso, y de algún nodo condició nado. De estas desmendradas condiciones, debe asumir la résponsa bilidad de los contenidos de su obra, sumándose a ello, la precariedad de medios económicos, las universidades ya no son generadoras de culture, y minimizan los presupuestos del mbro-la paulatina restricción y negación de canales representativos de participación y difusión, y la pemanente ocultación y manipulación de la realidad, que manejan los medios oficiales.

De estammanera el artista y su obra están sometidos a constantes presiones, y condenados a un progresivo aislamiento, que 10 : disocia de sus fuentes naturales de captación de la realidad, y por tanto, de una verdadera posibilidad de comunicación. 
En muchos de los comunicados elaborados por la UNAC, se hacía referencia a la noción de trabajadores del arte o trabajadores del arte y la cultura para agrupar las diferentes prácticas que no generen divisiones entre las diferentes formas entre visualidad, música, teatro, etc. Entre las declaraciones elaboradas por la UNAC, se hacían reflexiones sobre todo de la situación que les aquejaba y también del contexto represivo: "desarrollar libremente nuestras capacidades creadoras y [...] reclamar de la sociedad en que vivimos los medios para nuestra participación efectiva en la producción cultural" (UNAC, 1979). Ejemplo de ello se puede ver en un documento de 1979:

- Educación, frente a la privatización de las instituciones de educación que eleva los costos de aranceles e impide acceso, falta de participación democrática en la política educacional, falta de extensión universitaria a la comunidad que impide vincular ambas instancias.

- Censura, decretos que impedían el libre desarrollo de actividades culturales e importaciones de libros, revistas, junto con los impuestos aplicados a publicaciones y obras de arte, discos de música, así como listas negras que hay en medios de comunicación con nombres de creadores y sus obras.

- Cesantía, aquí se incluyen los despidos y exoneraciones de muchos artistas y creadores desde las universidades que estaban en manos de la dictadura, falta de reconocimiento y apoyo a actividades artístico-culturales, desaparición de compañías de teatro, galerías de arte, grupos musicales debido a la situación de represión y censura además de dificultades económicas.

- Limitaciones a los organismos gremiales, impedimentos para su funcionamiento e intervención del Ministerio del Interior en los asuntos propios de cada una de las asociaciones o de los colegios profesionales.

- Exiliados, ausencia de trabajadores culturales de reconocido prestigio, lo que no solo les afectaba como personas, sino también la circulación de su obra en el territorio nacional, en algunos casos no se autorizaba ingresar al país a creadores nacionales en gira.

- Detenidos desaparecidos, trabajadores de la cultura que estaban detenidos y desaparecidos a la fecha y que se hacía urgente e impostergable su liberación.

Entre otras actividades, la UNAC organizó un acto para el 75 aniversario del natalicio del poeta Pablo Neruda en el sindicato de trabajadores de la empresa Good Year en julio de 1979.

Frente a las opciones que hoy aparecen como únicas — concursos, becas, galerías, museos, etc.- la UNAC deberá gestar y entregar a los artistas, más alternativas de difusión y trabajo, que estén vinculadas con el pueblo: trabajos con sindicatos, con grupos culturales poblacionales, con parroquias, escuelas y otros, que revitalicen el quehacer artístico.

Frente a la disgregación producida por la represión, la Unidad por estos objetivos comunes.

Frente a la exaltación del trabajo individual, el trabajo colectivo; lo que no implica la anulación del trabajo individual, sino sumarnos —además—al trabajo común. (UNAC 1980) 
La UNAC también hizo parte del rechazo frente al plebiscito de 1980 en Chile en el que se aprobó la Constitución Política elaborada por la dictadura. En un documento titulado "A la opinión pública nacional," la UNAC señala que el texto constitucional fue elaborado a puertas cerradas por un grupo carente de representatividad nacional que además prolongaba el régimen dictatorial; indica que "el clima represivo, la falta de libertad de expresión y la ausencia de posibilidades de verificación popular tanto sobre este como sobre los otros actos de este gobierno, convierten este llamado en una farsa y en una insolencia que rechazamos y denunciamos con la mayor firmeza"; apelaba a que el trabajo en arte y cultura necesita participación social, posibilidades de diálogo y crítica; y hacía un llamado a los chilenos a rechazar "este simulacro y a seguir trabajando por una democracia verdadera".

\section{COORDINADOR CULTURAL}

El CC se funda en 1983, a diez años del golpe de Estado y en el contexto de las primeras Jornadas de Protesta Nacional. En medio de la implantación de las reformas y de los ajustes estructurales al modelo económico, político y cultural, se fortalece el movimiento de oposición a la dictadura en el que convergen sectores de trabajadores organizados, pobladores, estudiantes, el movimiento feminista y organismos de derechos humanos que confrontan públicamente al régimen mediante huelgas, partos y protestas territoriales. En medio de la ebullición, el CC fue un agente aglutinador del mundo cultural que dio continuidad a los esfuerzos iniciados por la UNAC a la vez que radicaliza su propuesta en la formación de un frente común de movilización y elaboración de una alternativa cultural para la lucha antidictatorial.

Un sector importante de los integrantes de la UNAC pasan a formar parte del CC y a la vez se suman actores emergentes del mundo artístico, sindical y poblacional. En las declaraciones y en los documentos públicos, firman más de cuarenta grupos vinculados al teatro, las artes visuales, el arte popular, la literatura, la música, la fotografía, los centros culturales, junto con organizaciones de trabajadores, derechos humanos y pobladores. La heterogeneidad de grupos que participaron del CC evidencia la potencia de una articulación que no se funda en afinidades estéticas o partidarias, sino en la transversalidad de una lucha que busca no solo producir un espacio de resistencia, sino también de alternativa respecto de la cultura dominante. Una alternativa que subvierte la jerarquía tradicional entre artistas más o menos institucionalizados, entre disciplinas, trayectorias, así como en la posibilidad de imaginar nuevos modos de relación entre la práctica artística y el quehacer político de manera extrainstitucional y sin sectarismos.

Uno de los rasgos que caracterizaron el accionar del CC fue la realización de intervenciones callejeras en lo que fue concebido como una disputa a la censura y el control sobre el espacio público. A través de acciones de arte en las que convocaron a actores, músicos y artistas visuales, realizaron múltiples acciones en el centro de la ciudad y en zonas de la periferia de Santiago para tomarse calles y plazas en las que estaba prohibida cualquier expresión de disidencia. El juego, la risa y la parodia, todas "estrategias de la alegría", como las Ilamara el artista conceptual y sociólogo argentino Roberto Jacoby, fueron herramientas importantes para que artistas y trabajadores de la cultura pudieran, como se plantea en un documento elaborado en 1988, "romper el cerco represivo, pero también, de luchar contra el escollo más difícil, la autocensura" (CC 1988, 1). 
Durante los tres años en que existió el CC, su principal gesta colectiva fue la realización del Congreso Nacional de Artistas y Trabajadores de la Cultura, para el que elaboraron un plan de despliegue por las distintas regiones del país donde se fundaron coordinadoras culturales locales, se realizaron precongresos y se invitó a la participación masiva. El Congreso, realizado los días 8, 9, 10 y 11 de diciembre de 1983 en la sede del sindicato de la Good Year y la Casa de Ejercicio San Ignacio de Loyola (CC 1983, 1), fue un hito fundamental en la configuración de un espacio de reflexión e imaginación política colectiva (figura 2).

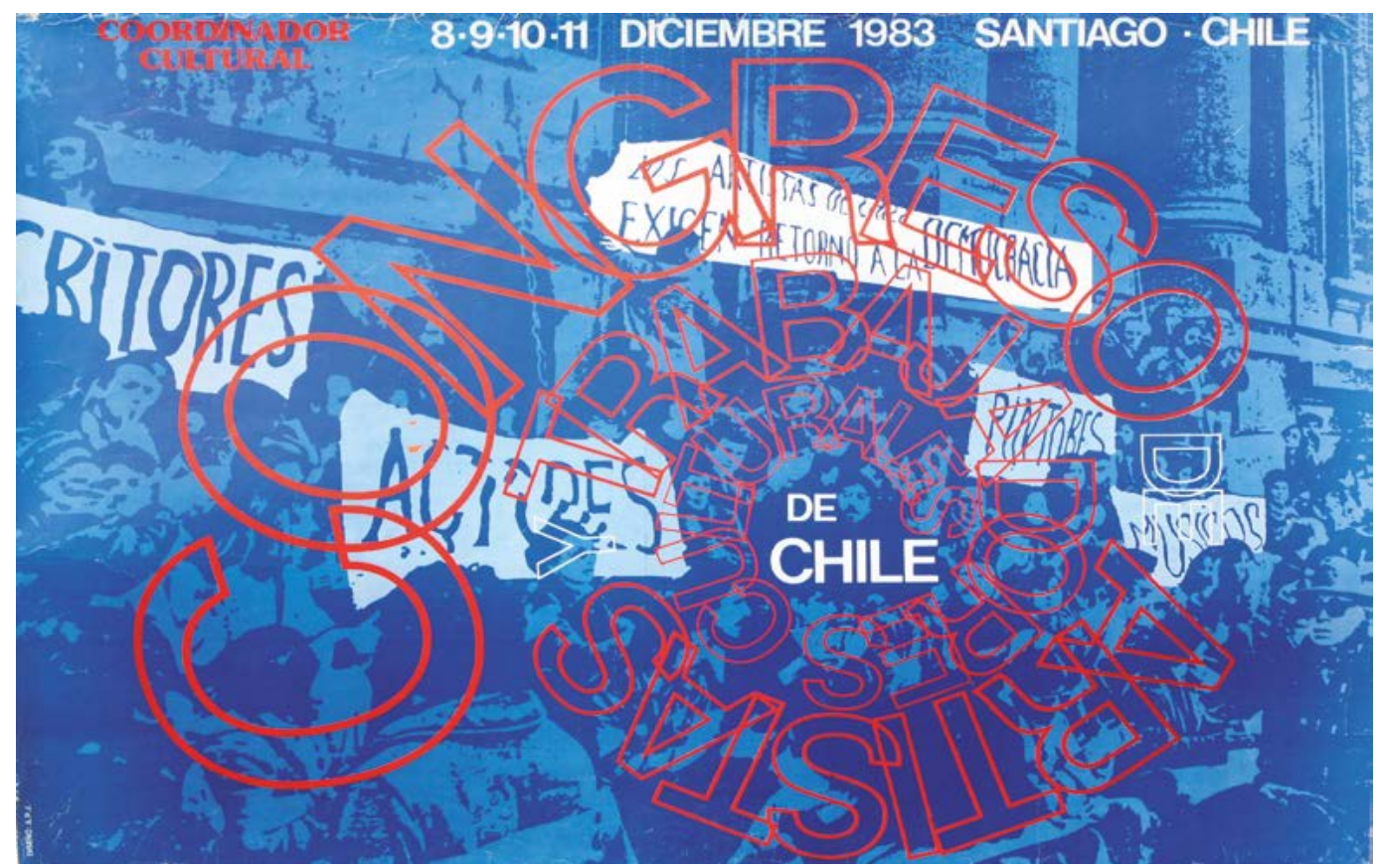

Figura 2. Afiche del Congreso Nacional de Artistas y Trabajadores de la Cultura. Fuente: Archivo Cucho Márquez, Santiago de Chile.

De manera absolutamente autónoma y sin apoyos institucionales, lograron realizar un congreso en el que participaron cientos de personas, entre ellas delegados internacionales. Durante estos días, se trabajaron distintos ejes temáticos, se realizaron exposiciones y presentación de obras teatrales y musicales.

En términos estructurales, el coordinador cultural estuvo conformado por una directiva en la que participaban representantes de distintos sectores de la cultura, particularmente de la literatura, la música, el teatro y las artes visuales. En su intención por descentralizar su marco de acción, el CC logró generar coordinadoras culturales locales, como es el caso de la ciudad de Valdivia donde se instalan con objetivos como el de "apoyar el desarrollo organizativo cultural en poblaciones y sindicatos de Valdivia" (Coordinador Cultural Valdivia 1984, 3) y también "elaborar una plataforma de reivindicativa de los artistas y trabajadores de la cultura local que busque, entre otras cosas, el derecho a ocupar los espacios institucionales y no institucionales de la ciudad" (3). Si bien en sus documentos suele referirse a la denominación del CC como estructura gremial (o sectorial), queda en evidencia que los objetivos y su ámbito de acción excede ampliamente el de demandas o defensas al mundo del arte, más bien se busca, junto con la defensa de las condiciones laborales de los trabajadores de la cultura, la conformación de un espacio cultural autónomo, crítico e imbricado en la lucha contra el régimen. La disputa cultural para el CC implica necesariamente asumir la idea de que "una cultura libre pasa primero por una sociedad libre, por un Chile sin Dictadura" (2). 
Así es como no existe la posibilidad de escindir el plano cultural del político o, lo que es lo mismo, de autonomizar la esfera artística del contexto social en que se despliega. La necesidad de esta relación no se plantea, exclusivamente, en un sentido ilustrativo o representacional, sino más bien en la participación dentro de iniciativas que de todas las formas en que sea posible fomenten "actitud combativa y participativa ante la intolerable gravedad del atropello a los Derechos Humanos que vive nuestro país" (Coordinador Cultural Valdivia 1984, 5).

La ocupación cultural de espacios públicos, institucionales y extrainstitucionales fue uno de los logros del CC mediante múltiples acciones y actividades a lo que se suma la reflexión colectiva respecto del posicionamiento sobre el lugar de la cultura y de las artes en el contexto dictatorial. En sus actas, documentos de convocatoria y publicaciones regionales, queda el rastro de sus discusiones, diagnósticos colectivos y proyecciones sobre el lugar de la cultura en un contexto de represión. En el documento con el que convocan al Congreso, plantean:

\footnotetext{
Por ello artistas y trabajadores de la cultura de Chile, nos autoconvocamos a efectuar en conjunta esta búsqueda de una política cultural que nos permita conducir nuestro trabajo hacia un futuro de libertad y democracia, y a concebir las acciones que podrán realizarla. A concentrarnos en torno a los elementos comunes que nos unen como trabajadores del arte, sin pretender legislar o dictaminar normas para encontrarnos en una generosa pluralidad estética para un mismo objetivo ético de participación activa y creadora en el proceso de construcción de una sociedad y una cultura nuevas para Chile. (Coordinador Cultural, 1983, 2)
}

Sin intentar neutralizar u omitir sus diferencias, la posibilidad extrainstitucional de reunir a grupos profundamente diversos, evidencia la potencia política de un espacio que no solo reclama un posicionamiento común frente a la macropolítica, sino que a la vez, y a contrapelo de la política dominante, elabora una micropolítica en la que prima el resguardo de la diferencia y la participación en común.

\section{COORDINADOR DE GREMIOS DEL ARTE}

EI CGA se funda en 1985 y pasa a ser la tercera y última de las grandes articulaciones culturales de la oposición a la dictadura cívico-militar. Desde un comienzo, el CGA, y en especial a partir de la organización de lo que sería el Segundo Congreso de Trabajadores de la Cultura, se concibe como una "continuidad organizativa del sector que está desde los primeros intentos post golpe por aglutinar al frente de la cultura" (Consultivo Coordinador de Gremios del Arte 1986, 1). Se piensa, en este primer sentido, como la extensión no solo de lo realizado por el CC o la UNAC, sino más ampliamente por las distintas iniciativas de resistencia cultural desde 1973. No obstante, es posible identificar en el CGA elementos que lo distinguen de las coordinadoras culturales anteriores y que instalan una preocupación por prefigurar la institucionalidad cultural futura. Esto último deja en evidencia el carácter contextual de sus reflexiones, puesto que se desarrollan, precisamente, en el momento de recomposición de los partidos de centro e izquierdas hacia el plebiscito. Continuidades y rupturas respecto de las anteriores coordinadoras culturales que determinarán particularmente los cambios en su composición, sus formas de construcción colectiva y los horizontes políticos y culturales que las guían. 
El CGA, tal como establece su nombre, da un paso más hacia la gremialización de la política cultural, al menos en lo relativo a la definición pública de su acometido. Si bien en sus declaraciones aún existe el ímpetu por la articulación cultural con miras a la movilización social, aparece de manera explícita la conformación de un frente cultural que genere un programa con vistas a la posdictadura. El cambio de nombre evidencia este giro de un proceso instituyente, como el que fuera el contexto de movilización y ebullición callejera, al de instituir una federación de trabajadores del arte que se ajuste a un futuro Gobierno democrático. La participación de partidos políticos se hace más presente hasta el punto de que uno de los objetivos declarados para la organización del Segundo Congreso de Artistas y Trabajadores de la Cultura sea "desarrollar los mecanismos para que los partidos políticos puedan formular sus respectivas propuestas sobre políticas culturales y su relación con un futuro proyecto de sociedad" (CGA 1986, 2). A diferencia de las coordinadoras culturales anteriores, que apelaban a la necesidad de la organización en un sentido general, el CGA convoca a los artistas y trabajadores de la cultura a integrarse a los organismos gremiales de su sector para desde ahí apoyar las iniciativas de movilización por sus derechos laborales y políticos. Resulta interesante considerar que la gremialización de la política fue una de las aspiraciones del proyecto cultural de la dictadura, en la medida en que solo los gremios estaban autorizados como entidades de organización tras reivindicaciones parcializadas y económicas. A pesar de que exista un giro a la noción de gremio de arte, en los documentos del CGA prevalece una mirada extensiva y politizante de los gremios que contrasta con la idea neutral y fragmentaria de los gremios oficialistas (figura 3).

La propuesta del CGA contempló una plataforma reivindicativa común de siete puntos:

1. Elaboración de una política cultural con participación de los artistas y trabajadores del área

2. Establecimiento de una previsión única para el sector

3. Abolición del IVA y todo gravamen a las artes y la cultura

4. Defensa de la libertad de creación y expresión

5. Términos de las "listas negras"

6. Defensa permanente de los Derechos Humanos

7. Defensa del patrimonio cultural de la nación. (CGA 1986, 2)

Siguiendo el marco gremial del CGA, disminuye considerablemente la composición y la diversidad de sus integrantes, dado que ya no participan colectivos, talleres o grupos, sino exclusivamente macroestructuras gremiales, tal como Sociedad de Escritores de Chile (SECH), Asociación de Pintores y Escultores de Chile (APECH), Sindicato de Actores de Radio, Teatro y Televisión (Sidarte), Asociación Gremial de Profesionales y Técnicos Audiovisuales (APTA), Asociación de Fotógrafos Independientes (AFI), Agrupación de Plásticos Jóvenes (APJ) y Colectivo de Escritores Jóvenes (CEJ).

Respecto del posicionamiento colectivo del CGA, el escultor Lautaro Labbé, quien lo presidió desde su fundación, plantea una mirada sobre la producción cultural como una herramienta de resistencia a lo largo de la dictadura y que en el presente se acerca a la posibilidad de acercarse al acometido de liberación del yugo dictatorial. Lejos de una definición 


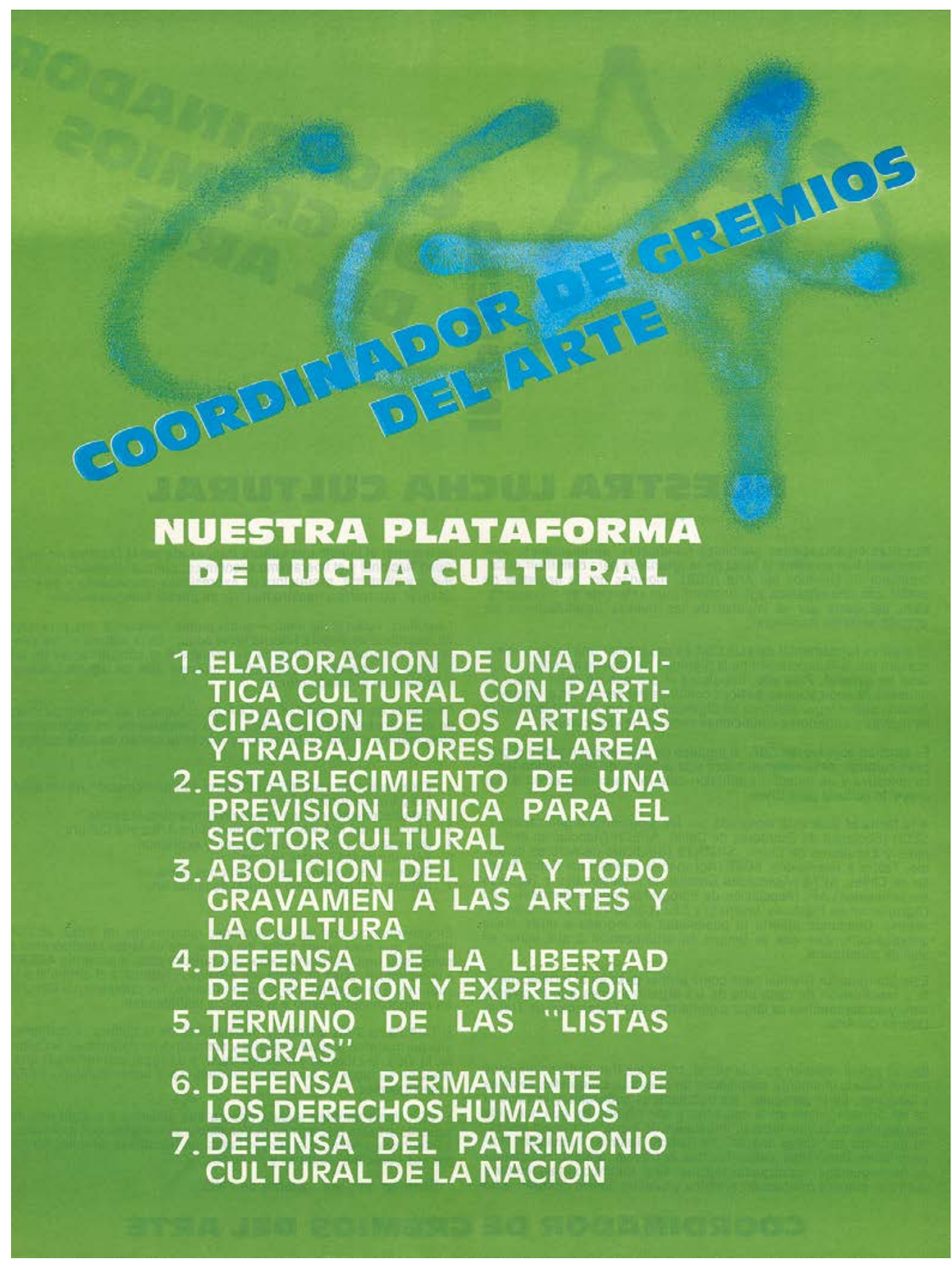

Figura 3. Afiche CGA.

Fuente: Archivo Luz Donoso, Santiago de Chile.

sustancialista o jerarquizante, la cultura aparece como un quehacer cotidiano, que se desenvuelve en la vida social junto con los espacios de creación y lucha popular. Serán precisamente estos los antecedentes del proceso de conformación de una alternativa que busque llevar a término la dictadura por medio de un proceso autorizado, institucional y transicional como es el plebiscito. La imaginación democrática, no es ya, como lo fuera en el CC, la experiencia de participación transversal desde la multiplicidad de agencias implicadas, sino la posibilidad concreta de asumir la cercanía del horizonte democrático. 


\section{MULTIPLICIDAD, OPOSICIÓN Y TRANSVERSALIDAD DE LAS COORDINADORAS CULTURALES}

Las tres generaciones de coordinadoras culturales fueron formando tramas de complicidad, articulación y acción colectiva que se desenvolvieron desde la oposición al régimen dictatorial desde 1977 hasta 1989. Todas son ejemplos de lo que Williams (1994) denomina "formaciones de oposición" en tanto comprenden su quehacer en la vía de una lucha más amplia que incluye a otros sectores sociales hacia una transformación social general. Las prácticas artísticas y culturales son un espacio de acción y una herramienta que posibilita la regeneración del tejido social, la ocupación del espacio público y se inscribe en las luchas de la memoria frente a la imposición del olvido. Junto con ello, se observa en las tres la presencia de elementos que coinciden con una "formación alternativa" en que lo que existe no es solo la resistencia a la cultura dominante, sino también el levantamiento de espacios, instancias y publicaciones que posibilitan la presentación y circulación de obras e intervenciones político-estéticas fuera del marco institucional.

Los modos de organización de las coordinadoras culturales entraman la experiencia militante de muchos de sus integrantes, pero también el deseo de crear nuevas formas de articulación colectiva. Si bien existían mesas ejecutivas y directorios que organizaban las dinámicas internas, los documentos y testimonios señalan que los roles asumidos por personas representantes de las organizaciones miembros, en general, fueron instancias que eran más bien de ejecución de actividades públicas, pero no se trataba de modelos jerárquicos. Además se hacía hincapié continuamente en la necesidad de la democracia interna y en la rotación de los roles cada ciertos periodos. Un rasgo común en todas las coordinadoras es que estaban ideadas bajo un principio de cuidado por la diferencia y la heterogeneidad interna, existía el deseo de reunir diferentes voces, diferentes prácticas, desde lugares de la ciudad que demostraran intereses situados en sus contextos. Asimismo, es importante destacar la necesidad de extender estas experiencias fuera de Santiago, el eje metropolitano, y desplazarse a diferentes ciudades de Chile, como fue el caso de la UNAC y el CC.

\section{CONCLUSIONES}

Las coordinadoras culturales no fueron solo espacios de discusión o articulación opositora, sino que también fueron polos de creación, experimentación y acción colectiva en el espacio público. Lejos de un "apagón cultural", lo que evidencia la existencia de estas coordinadoras culturales es la presencia de una red subterránea que existió a contrapelo de la cultura oficial y donde existió la experiencia de entidades porosas, situadas y múltiples, que en sus transformaciones y derivas evidencian los ciclos políticos y sociales de los que formaron parte.

La UNAC, el CC y el CGA son tres generaciones de articulaciones culturales que responden a cada contexto del periodo dictatorial. La UNAC (1977-1983) responde de manera intensa a la necesidad de agrupamiento en medio de la censura, la autocensura y el miedo instalado por políticas de control y eliminación. En medio del periodo fundacional de la dictadura cívico-militar en que se implantan las principales modificaciones al modelo de desarrollo hacia el neoliberalismo, la UNAC se propone regenerar el tejido social desde el mundo cultural, transversalizar la politización de las artes en todas sus expresiones y participar activamente 
en las primeras formas de movilización popular, particularmente en las huelgas del sector sindical. En segunda instancia, el CC (1983-1985) emerge en medio de la ola de protestas y manifestaciones de la oposición. Es el momento de mayor actividad pública de los distintos sectores organizados contra el régimen en lo estudiantil, lo sindical y lo poblacional, desde los organismos de derechos humanos, desde el movimiento feminista y desde la cultura. Las posibilidades que trae esto le permiten organizar el Primer Congreso de Artistas y Trabajadores de la Cultura, que será uno de los principales hitos de la resistencia cultural en dictadura. La radicalización de esta coordinadora respecto de la anterior es resultado de un agenciamiento colectivo que la llevó a desplegarse a nivel nacional en busca de generar un frente común de resistencia cultural. Finalmente, el CGA es el último estadio de las coordinadoras culturales en dictadura y también aquel en que los cambios en las estrategias de lucha política desde la insurgencia de masas a la vía institucional tuvo consecuencias en la articulación cultural. Por ello, de las tres coordinadoras culturales, esta sea la única que se plantea desde el punto de vista de una organización gremial y que a su vez asuma la necesidad de elaborar un programa cultural para la posdictadura. La democracia en este último caso aparece no solo como parte de la micropolítica de su organización y la disputa general por la recuperación democrática, sino como un horizonte macropolítico del que tienen que hacerse cargo. En este último caso, la imaginación política asume el papel de elaboración crítica desde lo extrainstitucional hacia lo institucional. En esta trayectoria queda en evidencia en cada caso, las líneas de continuidad, tal como el compromiso con la lucha antidictatorial y la concepción de una articulación que no distinguiera entre jerarquías artísticas, estéticas o políticas.

Hoy en día, existen diferentes exposiciones y proyectos de investigación nacionales e internacionales que buscan repensar el rol de los artistas desde el punto de vista de las formas de colaboración y prácticas artísticas en vínculo con comunidades. Muchas veces los referentes para estudiar estas experiencias provienen del contexto europeo o estadounidense, sobre todo por la falta de referentes no solo chilenos sino latinoamericanos. Proponemos que el estudio del material documental de la experiencia de las coordinadoras culturales en Chile sea una entrada situada de referencia para artistas e investigadores, porque tales experiencias históricas dan cuenta de estas formas de vincular los procesos de resistencia con exploración estética colectiva. Junto con ello, abre espacio a la genealogía de las prácticas de colaboración artística interdisciplinar, mucho antes de que fueran concebidas en esos términos.

El impacto que tuvo en las trayectorias individuales de los artistas haber formado parte de estas coordinadoras culturales fue muy significativo. En este sentido, la investigación sobre las coordinadoras culturales entrega pistas muy importantes para comprender la obra y el recorrido de artistas destacados, tales como Virginia Errázuriz, Lotty Rosenfeld, Luz Donoso, Lautaro Labbé, Raúl Zurita, Antonio Kadima, Havilio Pérez, Alberto Díaz Parra, Cucho Márquez, Janet Toro, entre muchos otros. Un estudio en profundidad sobre su participación en estas instancias permitiría analizar la influencia que esto tuvo en sus experiencias simultáneas y posteriores como artistas, a la vez que permitiría rastrear las claves de su participación creativa en las acciones colectivas. Sin duda, resulta fundamental contemplar un enfoque que ponga en relevancia la participación de las mujeres, tanto de aquellas que ocuparon posiciones visibles dentro de las coordinadoras culturales como de aquellas que no. La investigación muestra desde ya que, al igual que en la potencia del movimiento feminista de la década de 1980, en estos espacios de articulación y colaboración las mujeres ocuparon un papel fundamental en la posibilidad de atravesar y subvertir fracturas, fracciones y sectarismos en el campo cultural. 
La relevancia de recuperar la memoria de experiencias de articulación en el contexto dictatorial y que fueron capaces de imaginar y experimentar otras formas de hacer cultura, arte y política en común atiende, al igual que lo hicieron las propias coordinadoras culturales en su momento, a un deseo y a una necesidad de transformación absolutamente vigentes.

\section{NOTAS}

1. El periodo de la dictadura se inicia el 11 de septiembre de 1973 con el golpe cívico militar liderado pro Augusto Pinochet, que derrocó el Gobierno de la Unidad Popular (UP) presidido por Salvador Allende, quien perdió la vida ese mismo día. Este periodo se extendió durante diecisiete años, hasta que el plebiscito del 5 de octubre de 1988, cuando gana el no, detiene la continuidad de Pinochet en el poder. En 1989, se desarrollan candidaturas presidenciales, tras lo cual resulta electo Patricio Aylwin, quien tomó el poder en 1990, y así comenzó un proceso de transición democrática en Chile.

2. Para la exposición Poner el cuerpo: llamamientos de arte y política en América Latina en los años ochenta, de la que fueron curadoras ambas autoras, realizada en el Museo de la Solidaridad Salvador Allende en 2016, se pudo hacer un primer ejercicio de contextualización del escenario cultural de la década de 1980 y las coordinadoras culturales. Mediante un seminario internacional, mesas de debate con protagonistas de estos eventos y una residencia de investigación, se pudo tener un primer acercamiento a lo que implicó la UNAC, el CC y el CGA (Red Conceptualismos del Sur 2016).

3. Este artículo forma parte de la investigación "Coordinadoras culturales en dictadura" financiada por el FONDART de Investigación en Artes Visuales 2019.

\section{REFERENCIAS}

Amigo, Roberto et al. 2012. Perder la forma humana: una imagen sísmica de los años ochenta en América Latina. Madrid: Museo Nacional Centro de Arte Reina Sofía.

Aguiló, Osvaldo. 1983. Plástica neovanguardista, antecedentes y contextos. Santiago de Chile: Ceneca.

Álvarez Caselli, Pedro. 2004. Historia del diseño gráfico en Chile. Santiago de Chile: Consejo Nacional de la Cultura y las Artes.

Brunner, José Joaquín. 1979. La estructura autoritaria del espacio creativo. Santiago de Chile: Facultad Latinoamericana de Ciencias Sociales.

— 1985. La cultura como objeto de políticas. Santiago de Chile: Facultad Latinoamericana de Ciencias Sociales.

CC (Coordinador Cultural). 1983. Convocatoria al Congreso de Artistas y Trabajadores de la Cultura de Chile. Santiago de Chile: Coordinador Cultural.

Coordinador Cultural de Valdivia. 1984. Boletín N. ${ }^{\circ}$ 1. Valdivia: Coordinador Cultural.

Coordinador de Gremios del Arte. 1986. Consultivo Coordinador de Gremios del Arte. Trabajo comisión congreso de la cultura. Santiago de Chile: Coordinador de Gremios del Arte.

- 1989. Resistencia cultural desarrollada en estos 16 años de lucha contra la dictadura. Santiago de Chile: Coordinador de Gremios del Arte.

Cristi, Nicole y Javiera Manzi. 2016. Resistencia gráfica. Dictadura en Chile: APJ-Tallersol. Santiago de Chile: LOM.

Deleuze, Gilles y Guattari, Félix Mil mesetas. Madrid: Pre Textos, 1980

Donoso Fritz, Karen. 2013. “El 'apagón cultural' en Chile: políticas culturales y censura en la dictadura de Pinochet 1973-1983". Outros Tempos: Pesquisa em Foco-História 10 (16): 104-129. http://dx.doi. org/10.18817/ot.v10i16.285 
Errázuriz, Luis Hernán y Gonzalo Leiva Quijada. 2012. El golpe estético: dictadura militar en Chile, 19731989. Santiago de Chile: Ocho Libros.

Raunig, Gerald. 2007. "Revoluciones moleculares y prácticas artísticas transversales". Brumaria 8:234-237.

Red Conceptualismos del Sur. 2016. “EXPOSICIÓN: Poner el cuerpo. Llamamientos de arte y política en los años ochenta en América Latina". Consulta: 20 de enero de 2019 https://redcsur.net/es/2016/03/31/ exposicion-poner-el-cuerpo-llamamientos-de-arte-y-politica-en-los-anos-ochenta-en-america-latinamuseo-de-la-solidaridad-salvador-allende-santiago-de-chile/

Richard, Nelly. 1986. Márgenes e instituciones: arte en Chile desde 1973. Santiago de Chile: Art and Text/ Francisco Zegers.

Rivera, Anny. 1983. Transformaciones culturales y movimientos artísticos en el orden autoritario: Chile 1973-1982. Santiago de Chile: Ceneca.

UNAC (Unión Nacional por la Cultura). 1979. Llamado II. Santiago de Chile: UNAC.

— 1980. Informe comisión "libertad de expresión-libertad de creación."Área plástica. Santiago de Chile: UNAC.

— 1980 A la opinión pública nacional. Santiago de Chile: UNAC.

Vercauteren, David, Olivier Mouss Crabbé y Thierry Müller. 2010. Micropolíticas de los grupos para una ecología de las prácticas colectivas. Madrid: Traficantes de Sueños.

Williams, Raymond. 1994. Sociología de la cultura. Barcelona: Paidós. 